\title{
An Innovative Self-Feed Product For Elderly Arthritis Patients With Hand Disability
}

\author{
Hafeezur Rahmaan \\ Mohd Yassin \\ Universiti Sains Malaysia, \\ hafeezur@usm.my
}

\author{
Ahmad Zuhairi Abdul \\ Majid \\ Universiti Sains Malaysia, \\ zuhairi.majid@usm.my
}

\author{
Mohd Asyiek Mat Desa \\ Universiti Sains Malaysia, \\ asyiek@usm.my
}

\author{
Wong Pei Jun \\ Universiti Sains Malaysia, \\ peijun122@gmail.com
}

\begin{abstract}
Arthritis is a chronic disease, which affects the joints, tissues, organ and the body's internal system. Arthritis is the leading cause of disabilities and impaired quality of life. Besides, arthritis caused difficulties in carrying out daily activities independently. This study aims to innovate a product for elderly with arthritis in hand to self-feed at mealtimes. The design will enhance the independence or partial independence of arthritis elderly with hand disability at mealtimes. In the same time, the design will help to reduce the arthritis finger and hand pain experienced while eating. In addition, this study focuses on the challenges and difficulties faced by the elderlies with arthritic hand at mealtimes.

Keywords: Arthritis, elderly, self-feed, disability, product
\end{abstract}

\section{INTRODUCTION}

According to Disabled Living Foundation, eating and self-feeding are activity that people infrequently consider in detail; however, it is a core part of everyday living. When eating become difficult to do for a person, the problems can seem to be unduly dominant. People with hand disabilities need adaptive utensils to help them in self-feeding as they loss their grip strength.

Arthritis is a worldwide disease for elderly. According to the World Health Organisation, WHO (2015), five in 1,000 Malaysians are suffer from this disease (FMT News, 2015). Arthritis refers to joints inflammation; it can cause stiffness, pain, weak and deformity in joints (Merivale Hand Clinic, 2018). When arthritis affects the joints of the hands, it would rationalization stiffness and pain to the person (Halls et al., 2017). That pain can get worse whenever you use the hand a lot for repetitive tasks. For instance, gripping utensils in the kitchen or typing on a computer keyboard can make distress to the person. Besides, you may also lose strength in your hands and the weakness in your hands can make it hard to do even the simplest daily tasks, such as gripping cutlery, opening jars, writing and so on.

Severe arthritis can result in chronic pain, inability to do daily activities and make it hard to walk or climb stairs. Arthritis can cause permanent joint changes. Over time, joints can lose strength (Arthritis Foundation, 2016). Hand weakness person unable to grip properly. A variety of activities were reported to be difficult to do by them, with the two most common reported, eating with utensils and work activities that involving grasping or lifting, such as typing and writing (Stoppler, 2017). For a normal adult, lifting a spoon or a fork to mouth may seem like very easy but it's an outof-reach luxury for some adults and elderly with hand disabilities.

\section{OBJECTIVES}

The objective of this study is to investigate the challenges of elderly arthritis patient with hand disability at mealtimes. Analyzing the self-feed difficulties of elderly arthritis patient while using the utensils will also part of the study that resulting a solution for designing an ergonomic and practical cutlery for of elderly arthritis patient specifically to hand disability.

\section{METHODOLOGY}

In this study, only qualitative method has been used for collecting data. The methods used are shown as below:

\section{i) Literature Reviews}

Study collecting data from reliable internet sources, news, books, reports and journals.

\section{ii) Interviews}

Personal interviews have been conducted with orthopedist in Penang General Hospital, psychology lecturer of USM, caregiver of Than Hsiang Welfare Association, Ms. Wan Ching Yuen, the manager of The Penang Home for the Infirm and aged, and elderlies with arthritic hands. 


\section{iii) Observations}

Observing the challenges and difficulties faced by the elderlies with arthritic hands while they selffeed using utensils during mealtimes.

\section{FINDINGS}

Summary of Interview.

After carried out the interviews with the experts, arthritis patients should be divided in two groups, which are inflammatory arthritis and degenerative arthritis. Inflammatory arthritis caused inflammation in joints and tissues due to the immune system in body whereas degenerative arthritis breakdown the cartilage due to ageing. Inflammatory arthritis is known as rheumatoid arthritis and degenerative arthritis is referred to osteoarthritis. Some arthritis patients have limited range of motion in knee, ankle and wrist. The patient will experience pain and stiffness in hands and fingers whereas some of them facing the problem of deformity in hand and cause the patients unable to bend their fingers. They have to hold or grasp things which are bigger in size to prevent additional pain on fingers and hands.

\section{Summary of Observation}

Observation at The Penang Home for the Infirm and Aged.

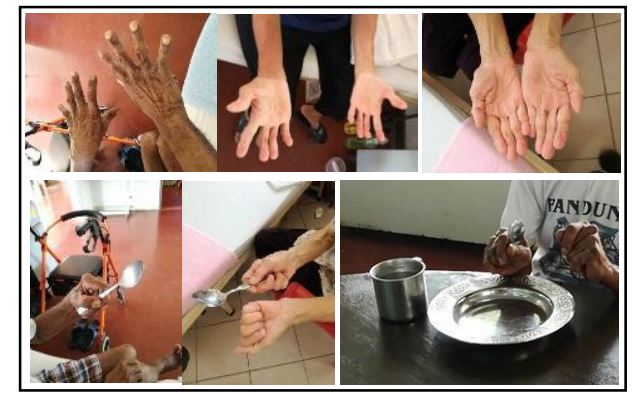

Figure 1.0 : Photos of arthritic hands and photos of utensils used by elderly arthritis patients

Photo taken: 13 April 2018

From the observations, elderly arthritis patient difficult to grip small objects properly as arthritis caused stiffness, pain, swollen and deform on their hand. However, they are able to self-feed at mealtimes but sometimes felt pain on fingers.

The cutlery used by the elderlies is just normal cutlery which is commonly found in market now. Basically, the form of the cutlery handle is flat dimple, flat straight or round dimple shaped. The form and the size of this kind of cutlery is not suitable for arthritis patients to use. This is because the size of the handle considered small for elderly with arthritic hand. Large handled cutlery is easier for them to grip as some of them comes with joints swelling, joints deformities make them unable to grip or grasp objects properly, especially small objects.

\section{Outcomes}

The outcomes from the interviews and observations are stated as below:-

a) Cutlery with larger handle

Cutlery with larger handle easy for arthritis patients to grip and reduce their hand and fingers pain while eating.

\section{b) Cutlery with strap}

Cutlery with strap help to support the hand or fingers of arthritis patients who have weak grip strength.

\section{c) Cutlery with twisted shaft}

Cutlery with twisted shaft is ideal for arthritis patients with limited range of motion of wrist.

\section{d) Bendable/flexible}

Bendable cutlery helps arthritis patients to ensure correct positioning of the cutlery during mealtimes.

\section{e) Soft material}

Soft material would not cause additional pain or injury to arthritis patients

\section{f) Comfortable}

The shape of the handle should be comfortable to grip so it would not cause additional pain and injury.

\section{DESIGN CONCEPT}

In this study, the design concept of the innovative adapted cutlery is come from magnetic two-way screw driver and mini flexible tripod. These two objects lead to designing and developing the idea, shape and function of the adapted cutlery.
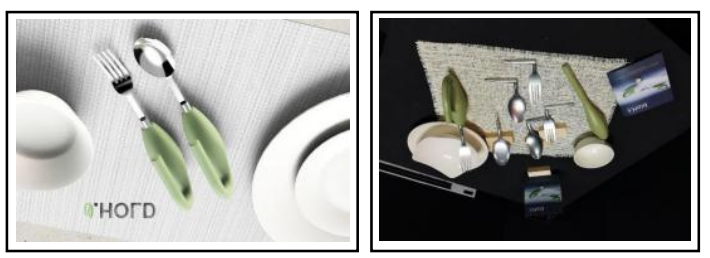

Figure 2.0: 3D Rendering Figure 3.0: Final Prototype

Big handle is designed for easy gripping whereas the shape of the handle follows human 
hand posture so that it is comfortable to hold. Furthermore, the attachments of the adapted cutlery are changeable. The twisted attachments are designed for arthritis elderlies with limited range of wrist motion to self-feed themselves.

\section{CONCLUSION}

This study is to identify the challenges and difficulties faced by the elderlies with arthritic hand during mealtimes. A study has been done through observations and interviews to identify the challenges and difficulties faced by the elderlies with arthritic hand during mealtimes.

There are some suggestions to enhance the features of the adapted cutlery. Firstly, the angle of the twisted shaft should be modified as the 90 degrees twisted shaft is not really suitable for the elderly arthritis patients with limited range of wrist motion. It might be too straight for the user while they are lifting the spoon or fork to the mouth. Further research is needed to find more about arthritic hand and more observations to find ideal angle for the shaft of the attachments. Another solution to address the problem is to design a shaft which can be bent according the user needs. Furthermore, the second suggestion is to add lock at the bottom part of the strap to prevent slip.

\section{REFERENCES}

Arthritis Foundation. (2016). What Is Arthritis? Retrieved from Understanding of Arthritis:

https://www.arthritis.org/about-arthritis/understandingarthritis/what-is-arthritis.php

FMT News. (2015, February 10). The curious case of rheumatoid arthritis. Malaysia. Retrieved from http://www.freemalaysiatoday.com/category/leisure /2015/02/10/the-curious-case-ofrheumatoidarthritis/

Halls, S., Dures, E., Kirwan, J. R., Pollock, J., Baker, G., Edmunds, A., \& Hewlett, S. (2017). Development and testing of candidate items for inclusion in a new rheumatoid arthritis stiffness patient-reported outcome measure. Rheumatology, 57(2), 263-272.

Merivale Hand Clinic. (2018). Retrieved from Arthritis: https://www.merivalehandclinic.co.nz/arthritis.php

Stoppler, C. (2017). Diseases and Conditions. Retrieved from

Arthritis: https://www.medicinenet.com/search/mni/arthritis8

World Health Organization. (2015). The Selection and Use of Essential Medicines: Report of the WHO Expert Committee, 2015 (including the 19th WHO Model List of Essential Medicines and the 5th WHO Model List of Essential Medicines for Children) (No. 994). World Health Organization. 